\title{
Error Recovery Based on FEC in Network-layer for Intermittently Connected
}

\section{Mobile Networks}

\author{
Yun $\mathrm{Li}^{1,2}$, Zhun Wang ${ }^{1}$, Xiaohu You ${ }^{2}$, Qi-lie Liu ${ }^{1}$, Mahmoud Daneshmand ${ }^{3}$ \\ ${ }^{1}$ Special Research Centre for Wireless Networks, Chongqing University of Post and Telecommunications, Chongqing 400065, \\ China \\ ${ }^{2}$ National Mobile Communications Research Laboratory, Southeast University, Nanjing, China \\ ${ }^{3}$ AT\&T Labs Research, Middletown, NJ, USA
}

\begin{abstract}
Reliable transmission in Intermittently Connected Mobile Networks (ICMNs) is a challenging work because the effective and reliable connection between the source and destination can not be sustained in ICMNs. To reliably hand over data to the destination, many dissemination-based routing protocols are proposed. Dissemination-based routing protocols assure nodes including intermediate nodes and destinations have more chances to receive packets, which will increase the probability that the packets can be correctly received by the destination. However, the existing error recovery mechanisms in network layer use the simple CRC to check the data packets independently, and discard the error packets even if one correct packet can be obtained from more than one partly error packet. In this paper, we propose a novel Network layer Error Recovery method based on FEC, named NER. NER divides a data packet into RS blocks and insert redundancy to each block. So the intermediate nodes and destinations can recover a correct data packet from multiple partially error copies of the same packet. The simulation results show that NER can obviously increase the delivery-ratio in ICMNs.
\end{abstract}

Keywords- Intermittently Connected Mobile Networks; error recovery; network layer; FEC;

\section{INTRODUCTION AND MOTIVATION}

Intermittently connected mobile networks (ICMNs) have broad application prospects in satellite communication networks, wireless ad hoc networks, sensor networks and military networks. ICMNs are characterized by: 1) Intermittent connectivity, there is no reliable end-to-end path between source and destination; 2) Long delay, long propagation delay and queuing delay significantly increase the end-to-end delay; 3) Asymmetric wireless link, the time-variable wireless channel makes the wireless link between two nodes have different data rate, even be unidirectional; 4) High error rate, interference and noise cause many bit errors on links.
The above characteristics make the traditional network architectures and protocols, for example the TCP/IP architecture, are not suitable for ICMNs. In recently years, some works have been conducted for these networks. The DTN (Delay Tolerant Networks) architecture is proposed by Delay Tolerant Network Research Group (DTNRG) of Internet Research Task Force (IRTF) when they study deep space Internet communications [1,2]. DTN uses the store-and-forward message switching to overcome the problems associated with intermittent connectivity and long delay.

One issue on ICMNs is to find the route to forward data packets. Routing techniques in ICMNs typically aim to maximize the delivery ratio or to minimize the delay that each message experiences during delivery [3]. Given the frequent topology dynamics that characterize ICMNs, both proactive and reactive routing approaches fail in finding appropriate routes for forwarding because they attempt to find complete paths between source and destination nodes, but these are not likely to exist. So it is more successful to find a route on a hop-by-hop basis, i.e., by searching the most appropriate next-hop node only once having traversed the hop before. A next-hop node is chosen by exploiting local information (also is called knowledge) about the contacts available at each hop towards other nodes as well as the queues of pending messages (waiting for forwarding) both at the forwarder node and at its neighbour nodes. In [4], the authors introduces four knowledge categories named oracles, contacts summary oracle, contact oracle, queuing oracle and traffic demand oracle, and proposes different routing algorithms, each of them using a different set of oracles. Unfortunately, the assumption of availability of the oracles is not actually realistic. So most of the existing routing protocols on ICMNs are not based on the knowledge about the future topology of the network, and dissemination-based routing, including replication-based routing protocols [5-8], coding-based routing[9,10], utility-based routing $[11,14]$, are proposed. The heuristic 
behind dissemination-based routing is that, since there is no knowledge of a possible path towards the destination node of a message, nor of an appropriate next-hop node, the message can be sent to everywhere. It will reach the destination node by passing node by node eventually. The main problem of dissemination-based routing is that a large number of copies of the same packet exist in the network, and will be received and forwarded by many nodes, which exhausts the wireless bandwidth resource, and wastes the energy of mobile node. Another problem about dissemination-based routing is that the copies received by the intermediate nodes and destinations, especially the partially error copies, are directly dropped without fully utilizing. To resolve the above problems, we propose a novel network layer error recovery method, named NER. NER utilizes the characteristic of dissemination-based routing in ICMNs, i.e., more than one copy can be received by the intermediate nodes and destinations, to recover a correct data packet from partially error packets, which can increase the delivery ratio of message in ICMNs.

Except the routing problem, error control and recovery is another challenging issue in ICMNs. Because of the intermittent connectivity, high bit error and unidirectional link, many packets will be corrupted, which makes many packets sent by source can not be received by the destination. Although many error recovery mechanisms have been proposed for traditional networks, including ARQ in link layer and transport layer, these mechanisms are based on the bidirectional link, short round-trip delay and reliable end-to-end connection, which are not suitable for ICMNs. Another error control method in networks is forward error correction (FEC). FEC can recover many errors by inserting redundant information to the packets. Although many FEC methods are proposed to improve the performance of wireless networks [15], most of works are in link layer or application layer. To our knowledge, this is the first time to use the FEC to recovery the error in network layer in ICMNs. And the dissemination-based routing make the error recovery based on FEC in network layer can be easily implemented.

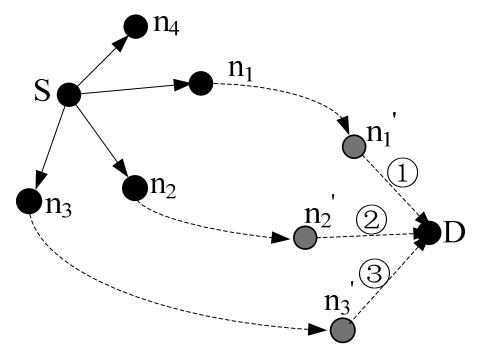

Figure 1. An example to show the motivation.
We give the motivation of this paper through an example in Fig. 1. In Fig. 1, at time $t_{0}$, the source node $S$ want to transmit data packet $\mathrm{P}$ to destination node $\mathrm{D}$. For the dissemination-based routing, $\mathrm{S}$ first delivers $\mathrm{P}$ to its neighbors at time $t_{0}$, which are $n_{1}, n_{2}$ and $n_{3}$. Let $n_{1}, n_{2}$ and $n_{3}$ can contact with $D$ at time $t_{1}, t_{2}$ and $t_{3}\left(t_{1}<t_{2}<t_{3}\right)$, respectively. Then $\mathrm{n}_{1}$ will first forward $\mathrm{P}$ to $\mathrm{D}$. We assume $\mathrm{P}$ forwarded by $n_{1}$ is corrupted because errors, then $D$ will drop it and wait the other nodes to forward P. In this example, $\mathrm{n}_{2}$ will forward $P$ to $D$ at $t_{2}$. If the packet forwarded by $n_{2}$ is error once more, D can correctly received $\mathrm{P}$ only if $\mathrm{n}_{3}$ successfully transmits $\mathrm{P}$ to D. However, if we use FEC in network layer, we can increase the probability that $P$ is successively transmitted by $n_{1}$ and $n_{2}$. And more importantly, even if the packets that destination $\mathrm{D}$ received from $\mathrm{nl}$ and $\mathrm{n}_{2}$ are corrupted, we can recover a correct packet form these two partially packet copies by using multi-copy recombining correction technique, which is proposed in the following section.

The above example motivates us to propose FEC in network layer to recover a correct data packet from multiple partially error copies of the same packet. The analysis results show that the proposed NER can increase the delivery ratio, and in turn save the bandwidth and energy consumption.

In Section II, we will describe the NER in details. The performance of NER is analyzed in Section III and Section IV. The conclusion is given in Section $\mathrm{V}$.

\section{ERROR RECOVERY IN NETWORKLAYER}

The error recovery in network layer based on FEC (NER) mainly includes two procedures, one is the procedure in the source node, and the other is the procedure in the intermediate nodes or destinations.

\section{A. The procedure in the source node}

The main function of NER in source nodes is to encapsulate messages in data packet, and divide each data packet into blocks. For each block, the redundant information will be added to check and correct the errors in the block.

Although there are many error correcting codes can be used to FEC, in this paper, we select Reed Solomon (RS) code to illustrate the procedures of NER and to analyze the performance of NER. Table I gives the several common RS codes [16]. For ICMNs, RS $(127,117)$ is more suitable because of the higher bit-rate coding, error-correcting capability and lower failure probability.

TABLE I. SERVAL COMMON CODES OF RS

\begin{tabular}{|c|c|c|c|}
\hline Type & Speed(k/n) & $\begin{array}{c}\text { Error } \\
\text { correcting } \\
\text { capability }\end{array}$ & $\begin{array}{c}\text { Fail } \\
\text { probability }\end{array}$ \\
\hline $\mathrm{RS}(511,479)$ & 0.93 & 16 & $5.78 \mathrm{e}-1$ \\
\hline
\end{tabular}




\begin{tabular}{|l|c|c|c|}
\hline $\operatorname{RS}(255,223)$ & 0.87 & 16 & $1.48 \mathrm{e}-1$ \\
\hline $\operatorname{RS}(127,117)$ & 0.92 & 5 & $3.48 \mathrm{e}-3$ \\
\hline $\operatorname{RS}(63,55)$ & 0.85 & 4 & $1.48 \mathrm{e}-4$ \\
\hline $\operatorname{RS}(31,25)$ & 0.81 & 3 & $1.03 \mathrm{e}-4$ \\
\hline
\end{tabular}

The detail procedure of NER using RS code in source node is shown in Fig.2. In Fig.2, the packet header is an independent block, and the payload is divided to $N$ blocks, each block is 127 octets including 117 octets data and 10 octets redundancy. When length of data part of the last RS block is shorter than 117 octets, complemental bits ' 0 ' will be added. RS $(127,117)$ code can correct up to 5 bytes errors or 10 bytes errors of known location[16].

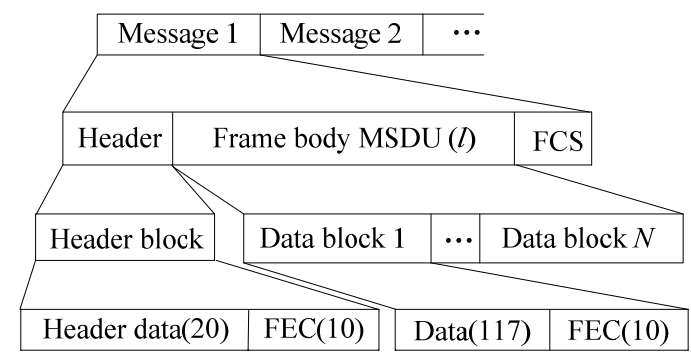

Figure 2. The procedure in the source node.

B. The procedure in intermediate nodes or destinations

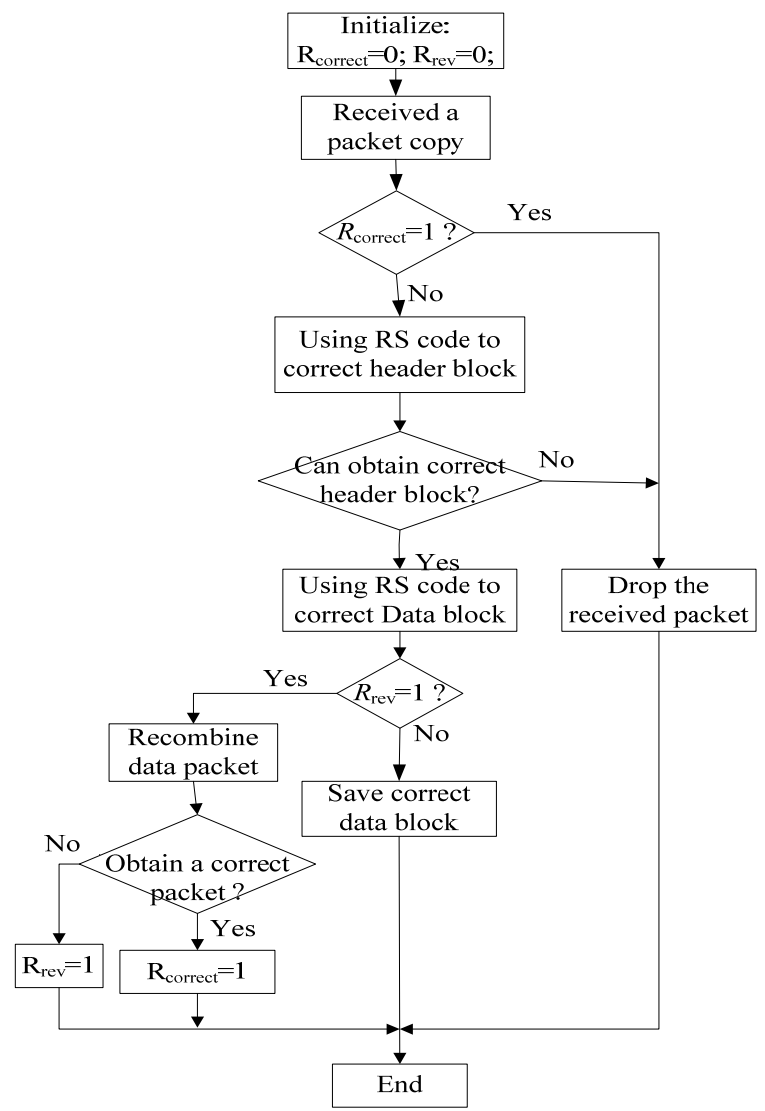

Figure 3. RS error correction and multi-copy resembling process

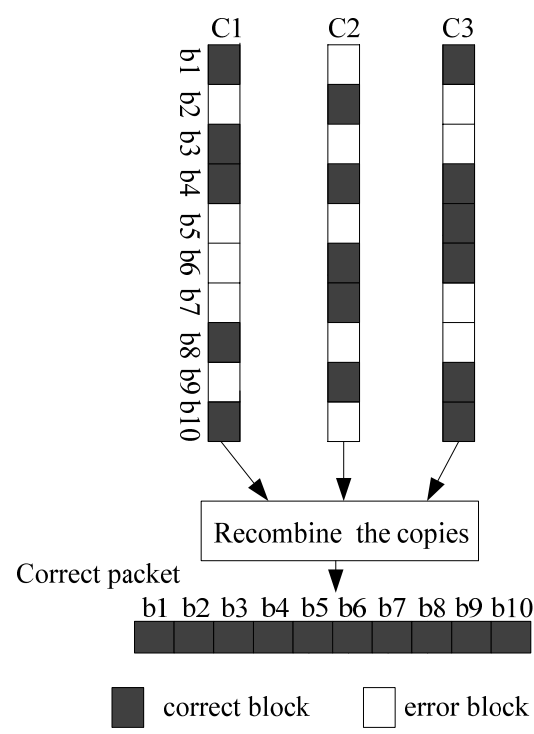

Figure 4. Multi-copy recombining process

The main function of NER in intermediate nodes or destinations is to recover a correct data packet from one or more corrupted copies of a packet. The process flow chart of NER in intermediate nodes or destinations is shown in Fig. 3, which mainly includes RS error correction and multi-copy recombining functions. In Fig.3, the variable $R_{\text {correct }}$ shows whether a correct copy of the same packet is buffered or not, and $R_{\text {rev }}$ shows whether a copy of the same packet is buffered or not.

After receiving a copy of data packet, the intermediate nodes or destinations use the RS error correction function to try to correct each block in that packet, and the correct blocks are buffered.

The multi-copy recombining is an important function, which is the main difference between NER and traditional network layer function. In traditional network layer, the corrupted packets will be wholly discarded. In NER, the correct blocks in a data packet will be buffered, and the different correct blocks in different copies of the same data packet will be recombined to try to recover a correct packet. Fig.4 shows an example of multi-copy recombining function, in which different blocks from $\mathrm{C} 1, \mathrm{C} 2$, and $\mathrm{C} 3$ are recombined. Here $\mathrm{C} 1, \mathrm{C} 2$, and $\mathrm{C} 3$ are three copies of the same packet $\mathrm{P}$.

\section{PERFORMANCE ANALYSIS}

In this section, we analyze the end-to-end error probability, which is defined as the probability that a packet transmitted by the source node is not correctly received by the destination. We will first calculated the physical (PHY) layer bit error probability for the AWGN channel, and deduce the RS-coded block error probability. Then, the 
failure probability of multi-copy recombining and the end-to-end error probability will be analyzed.

\section{A. PHY bit error probability}

We assume that the noise over the wireless medium is white Gaussian with spectral density $N_{0} / 2$. And believe that our error performance analysis based on the AWGN model will show the same trend as that based on a more realistic and complicated channel model.

For the rectangular constellation with $M=2^{k}$, the symbol error probability for an $M$-ary QAM is [17]:

$$
p_{M}=1-\left(1-P_{\sqrt{M}}\right)^{2}
$$

Where the $P_{\sqrt{M}}$ is error probability for the M-ary QAM with average signal-to-noise ratio $\varepsilon_{a v} / N_{0}$, which is given by:

$$
p_{\sqrt{M}}=2\left(1-\frac{1}{\sqrt{M}}\right) Q\left[\sqrt{\frac{3}{M-1} \frac{\varepsilon_{a v}}{N_{0}}}\right]
$$

The bit error rate (BER) for M-ary QAM with Gray coded constellation mapping can be approximated by:

$$
P_{b}^{(M)} \approx \frac{1}{\log _{2} M} \cdot P_{M}
$$

For BPSK modulation, the BER is the same as the symbol error probability, which is given by[17]:

$$
P_{b}^{B}=Q\left(\sqrt{2 \frac{\varepsilon_{a v}}{N_{0}}}\right)
$$

Where the $\varepsilon_{a v} / N_{0}$ is average signal-to-noise ratio.

\section{B. $\quad R S$ coded block error probability}

Let $P_{\mathrm{b}}$ be the bit probability of QAM or BPSK, $P_{\mathrm{o}}$ be the error probability of an Octet, which is equal to the probability that at least one bit in a Octet is error, then

$$
p_{o}=1-\left(1-p_{b}\right)^{8}
$$

Given a RS encoded block $\mathrm{RS}(\mathrm{n}, \mathrm{k})$, here $k$ is length of data and $n-k$ is the redundancy. The error probability of $\mathrm{RS}(\mathrm{n}, \mathrm{k})$ is given by [15]:

$$
p_{R S}(n)=1-\sum_{i=0}^{t}\left(\begin{array}{l}
n \\
i
\end{array}\right)\left(p_{o}\right)^{i}\left(1-p_{o}\right)^{n-i}
$$

Where $t=[(\mathrm{n}-\mathrm{k}) / 2]$.

\section{Failure probability of multi-copy recombining}

Suppose that the intermediate nodes or destinations receive $R$ copies of a packet, the probability that they can not reconstruct a complete correct packet is defined as the failure probability of multi-copy recombining, $P_{F}(R)$.

According to NER, only the copies whose header block can be corrected by RS will be used to recombine packet. Let $R$ ' be the number of copies with correct header blocks,
$C_{i}=\left\{B_{i j}, 1 \leq i \leq R^{\prime}, 1 \leq j \leq N\right\}$ denote the $i^{\text {th }}$ packet copy with correct header block, and $B_{i j}$ be the $j^{\text {th }}$ block of $C_{i}$. The $P_{F}(R)$ is given in Eq. (7) when adopted the multi-copy recombinant technology is given by:

$$
\begin{array}{r}
P_{F}(R)=\sum_{R^{\prime}=0}^{R}\left(\begin{array}{l}
R \\
R^{\prime}
\end{array}\right)\left(P_{H D}\right)^{R-R^{\prime}}\left(1-P_{H D}\right)^{R^{\prime}} . \\
{\left[1-\left(1-\left(P_{D B}\right)^{R^{\prime}}\right)^{N}\right]}
\end{array}
$$

Where $P_{H D}=P_{R S}(30)$ is the error probability of header block, $P_{D B}=P_{R S}(127)$ is the error probability of data block. $\left(P_{D B}\right)^{R}$ is the probability of recombining a correct data block from $R^{\prime}$ data blocks $\left\{B_{i j}\right.$, for a given $j$, and $\left.1 \leq i \leq R^{\prime}\right\} . \quad\left(1-\left(P_{D B}\right)^{R^{\prime}}\right)^{N} \quad$ is the probability of recombining a correct packet from $R^{\prime}$ copies with correct header.

\section{End-to-end error probability in given topology}

The end-to-end error probability $\left(P_{E}\right)$ is defined as the probability that a packet transmitted by the source node is not correctly received by the destination. In the following, we will analyze the $P_{E}$ for Line and Grid topologies which are shown in Fig. 5 and Fig. 6, respectively.

To increase the delivery ratio, we divide the payload of packet to blocks with 117 bytes, and use RS $(127,117)$ to encode each block. For packet with $l$ bytes payload, it can be divided into $N=\operatorname{ceil}(\mathrm{l} / 117)$ blocks.

In the line topology shown in Fig.5, a packet with $l$ byte payload sent by $\mathrm{S}$ can be received by $\mathrm{D}$ only if this packet can be successfully transmitted by each hop. So the $P_{E}$ can be calculated by Eq. (8).

$$
P_{E}=1-\left[\left(1-p_{R S}(30)\right)\left(1-p_{R S}(127)\right)^{N}\right]^{H}
$$

Where, $H$ is the hop count from $\mathrm{S}$ to $\mathrm{D}$.

On the other hand, the end-to-end error probability of a non-RS-coded packet with $l$ bytes can be approximated as:

$$
P_{E}^{\prime}=1-\left[\left(1-p_{b}\right)^{(20+l) \times 8}\right]^{H}
$$

In the Grid topology shown in Fig.6, we use dissemination-based routing to forward packet. So some intermediate nodes and destination will receive multiple copies of the packet sent by S. To determine which nodes will receive multiple copies of a packet and how many copies they can receive, we show the possible shortest paths between $\mathrm{S}$ and D in Fig. 7 when using dissemination-based routing.

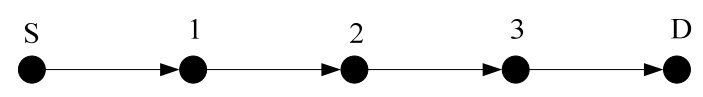

Figure 5. Line Topology. 


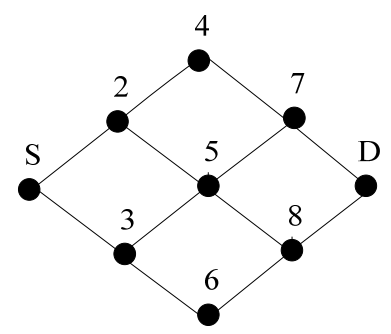

Figure 6. Grid Topology.

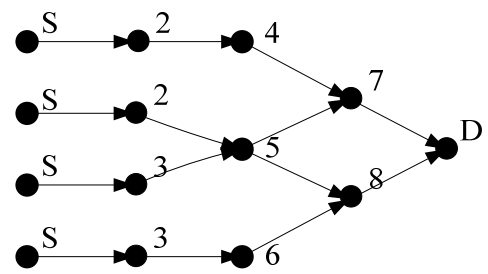

Figure 7. Shortest paths when using dissemination-based routing to Fig. 6

According to Fig.7, the node 5, 7, 8, and D can receive 2 copies of a packet.

Let the payload of packet is $l$ bytes, using RS-coded, the error probability at the node 2 and node 3 is:

$$
P_{2,3}=1-\left(1-p_{R S}(30)\right)\left(1-p_{R S}(127)\right)^{N}
$$

In node 5, we use multi-copy recombining technique because two copies from node 2 and node 3 can be received. So the error probability at the node 5 is:

$$
P_{5}=P_{2,3}{ }^{2}+2 P_{2,3}\left(P_{2,3}-P_{2,3}{ }^{2}\right)+\left(1-P_{2,3}\right)^{2} P_{F}(2)
$$

Similarly, we can obtain the error probability at the node 4, 6 as Eq. (12), and the error probability at the node 7, 8 as Eq. (13)

$$
\begin{gathered}
P_{4,6}=1-\left(1-P_{2,3}\right)^{2} \\
P_{7,8}=P_{4,6} P_{5}+\left(1-P_{5}\right) P_{4,6} P_{2,3} \\
+\left(1-P_{4,6}\right) P_{5} P_{2,3}+\left(1-P_{4,6}\right)^{2} P_{F}(2)
\end{gathered}
$$

Finally, the error probability and destination D, which is the end-to-end error probability $P_{E}$, is:

$$
\begin{aligned}
P_{E} & =P_{7,8}{ }^{2}+\left(1-P_{7,8}\right) P_{7,8} P_{2,3} \\
& +P_{7,8}\left(1-P_{7,8}\right) P_{2,3}+\left(1-P_{7,8}\right)^{2} P_{F}
\end{aligned}
$$

On the other hand, the end-to-end error probability of a non-RS-coded packet with $l$ bytes can be approximated as:

$$
P_{E}^{\prime}=\left\{1-\left[\left(1-p_{b}\right)^{(20+l) \times 8}\right]^{4}\right\}^{2}
$$

\section{NUMERICAL RESULTS}

In this section, we evaluate of NER by numerical method. To calculate the end-to-end error probability, the length of packet payload is 512 bytes, which can be divided to 5 data blocks. The size of each data block is 127 bytes, including 117 bytes data and 10 bytes redundancy. The end-to-end error probability of NER is calculated according to Eq. (8) and Eq. (14) for Line topology and Grid topology, respectively. The end-to-end error probability of non-RS-coded method is calculated according to Eq. (9) and Eq. (15) for line topology and Grid topology, respectively. And the numerical results are shown in Fig. 8 and Fig. 9. Fig. 8 and Fig. 9 show that NER can significantly decrease the end-to-end error probability.

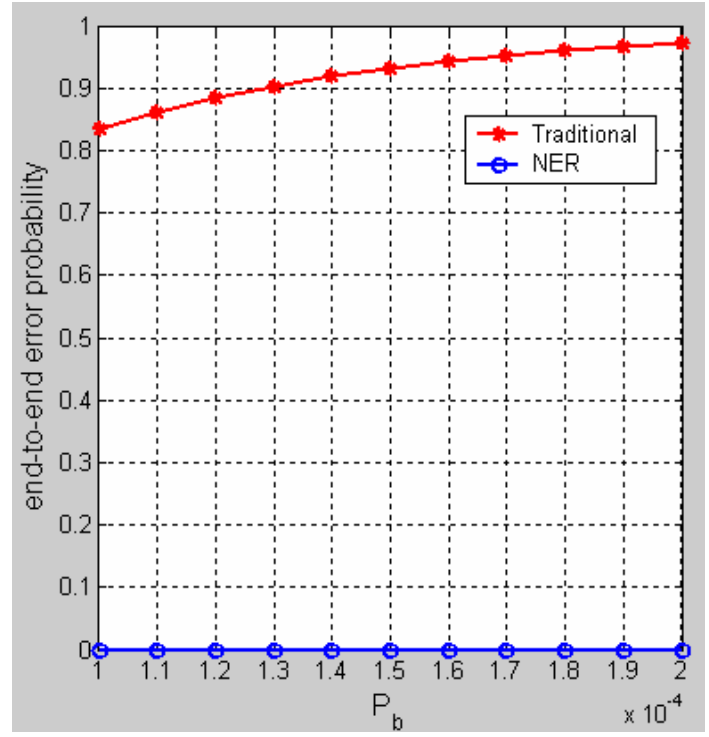

Figure 8. End-to-end error probability vs. different $P_{\mathrm{b}}$ for Line topology.

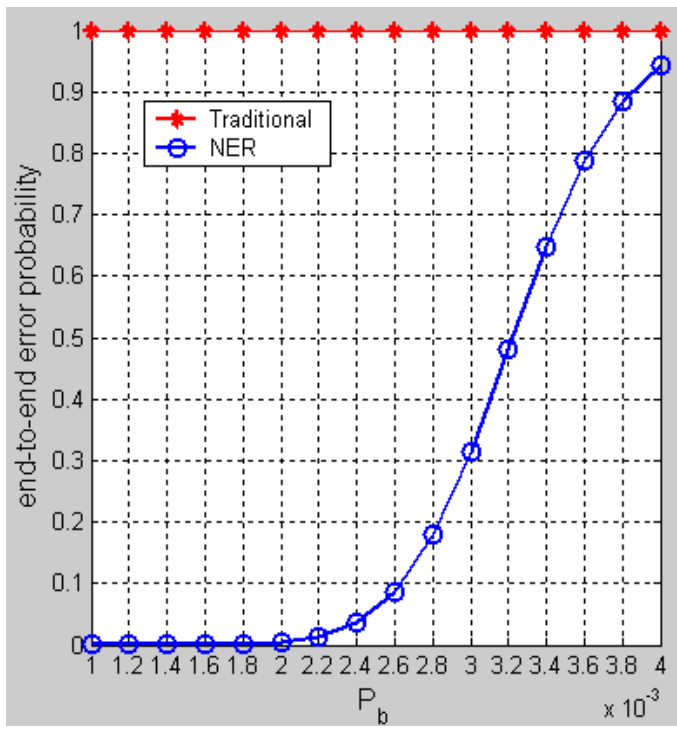

Figure 9. End-to-end error probability vs. different $P_{\mathrm{b}}$ for Grid topology. 


\section{CONCLUDING}

In this paper, we propose network layer error recovery for ICMNs, which is named NER. NER use RS code and multi-copy recombining scheme to improve the end-to-end performance of ICMNs. Although this paper select RS code, we believe that NER is efficient for other FEC codes.

In the future work, we will evaluate the performance more comprehensively.

\section{ACKNOWLEDGEMENT}

This paper is supported by the National Science Foundation of China $(60702055,61071118)$, Program for New Century Excellent Talents in University (NCET-07-0914), Natural Science Foundation Project of CQ CSTC, the Grant by the Science \& Tech. Commission of Chongqing (CSTC2009BB2279), and China Postdoctoral Science Foundation (20090451158).

\section{REFERENCE}

[1] Cerf V, Burleigh S and Hooke A, et al. Delay-tolerant networking architecture[EB/OL]. (2007). http//www.ipnsig.org.

[2] Fan Xiu-mei. Delay-tolerant networking architecture and key technologies research [J]. Electronic Journal vol.1. 2008, pp.47-55.

[3] L. Pelusi, A.Passarella, and M. Conti, "Beyond MANETs: Dissertation on Opportunistic Networking," IIT-CNR. Tech. Rep., May 2006

[4] J. Sushant,K. Fall, and R. Patra, "Routing in a delay tolerant network," in Proceedings of SIGCOMM'04,August,2004.

[5] Vahdat and D. Becker, "Epidemic routing for partially connected ad hoc networks", Tech. Rep. CS-2000-06, Department of Computer Science, Duke University, Durham, NC, 2000.

[6] Davids, A. H. Fagg, and B. N. Levine, "Wearable Computers as Packet Transport Mechanisms in Highly-Partitioned Ad-Hoc Networks", in Proceedings of the Inter-Symposium on Wearable Computing, Zurich, October, 2001.

[7] B. Burns, O. Brock, and B. N. Levine, "MV Routing and capacity building in disruption tolerant networks", in Proceedings of the IEEE
INFOCOM 2005, Miami, FL, March, 2005.

[8] T. Spyropoulos, K. Psounis, and C. S. Raghavendra, "Spray and Wait: An Efficient Routing Scheme for Intermittently Connected Mobile Networks", in Proceedings of the ACM SIGCOMM 2005 Workshop on delay tolerant networks, Philadelphia, PA, USA, August 22-26, 2005 .

[9] Y. Wang, S. Jain, M. Martonosi, and K. Fall, "Erasure Coding Based Routing for Opportunistic Networks", in Proceedings of the ACM SIGCOMM 2005 Workshop on delay tolerant networks, Philadelphia, PA, August 22-26, 2005.

[10] J. Widmer and J.-Y. Le Boudec, "Network Coding for Efficient Communication in Extreme Networks", in Proceedings of the ACM SIGCOMM 2005 Workshop on delay tolerant networks, Philadelphia, PA, USA, August 22-26, 2005

[11] X. Chen and A. L. Murphy, "Enabling disconnected transitive communication in mobile ad hoc networks", in Proceedings of the Workshop on Principles of Mobile Computing (collocated with PODC'01), Newport, RI (USA), pages 21-27, August,2001.

[12] Lindgren, A. Doria, and O. Schelèn, "Probabilistic routing in intermittently connected networks", Mobile Computing and Communications Review, 7(3), July, 2003.

[13] M. Musolesi, S. Hailes, and C. Mascolo, "Adaptive Routing for Intermittently Connected Mobile Ad Hoc Networks", in Proceedings of the 6th IEEE International Symposium on a World of Wireless, Mobile and Multimedia Networks (WoWMoM 2005), Taormina-Giardini Naxos, Italy, June 13-16, 2005.

[14] F. Tchakountio and R. Ramanathan, "Tracking highly mobile endpoints", ACM Workshop on Wireless Mobile Multimedia (WoWMoM), Rome, Italy, July, 2001.

[15] Zhu Xiao-gang, Yang Chuan-hou. FEC coding in a reliable Multicast Application Analysis [M]. Shanghai Jiaotong University Journal, Vol 33.1999. No. 5.

[16] Yuan qing. Mobile Ad-Hoc Network Data Reliable Transmission research [D]. National Defense Science and Technology University, 2007.

[17] John G. Proakis. Digital communication, 2001.10 